\title{
Contradictory responses of the microcirculation to changes in extracorporeal membrane oxygenation pump flow in patients on venoarterial extracorporeal membrane oxygenation: a prospective observational study
}

\section{Tzu-Jung Wei}

Department of Anesthesiology, National Taiwan University Hospital

\section{Chih-Hsien Wang}

Department of Surgery, National Taiwan University Hospital

\section{Wing-Sum Chan}

Far Eastern Memorial Hospital Department of Anesthesiology

\section{Yi-Chun Yeh}

National Taiwan University Institute of Epidemiology and Preventive Medicine

Chien-Heng Lai

National Taiwan University Hospital Department of Surgery

\section{Anne Chao}

National Taiwan Univsesith Hospital Department of Anesthesiology

Chi-Hsiang Huang

National Taiwan University Hospital Department of Anesthesiology

\section{Ming-Jiuh Wang}

National Taiwan University Hospital Department of Anesthesiology

\section{Ya-Jung Cheng}

National Taiwan Univsesity Hospital Department of Anesthesiology

\section{Yih-Sharng Chen}

National Taiwan University Hospital Department of Surgery

\section{Can Ince}

Erasmus MC Department of Intensive Care

\section{Tzu-Yu Lin}

Far Eastern Memorial Hospital Department of Anesthesiology

Yu-Chang Yeh ( $\nabla$ tonyyeh@ntuh.gov.tw )

National Taiwan University Hopistal https://orcid.org/0000-0001-5143-5520 
Keywords: Extracorporeal membrane oxygenation, Microcirculation, Cardiogenic shock

Posted Date: February 7th, 2020

DOl: https://doi.org/10.21203/rs.2.22931/v1

License: (c) (1) This work is licensed under a Creative Commons Attribution 4.0 International License. Read Full License 


\section{Abstract}

Background: Venoarterial extracorporeal membrane oxygenation (VA-ECMO) pump flow is crucial for maintaining tissue and organ perfusion in patients with cardiogenic shock. Determining the optimal VAECMO pump flow rate to maintain adequate tissue perfusion remains a clinical challenge. Poor microcirculation is associated with higher mortality in patients using VA-ECMO. Change in VA-ECMO pump flow is expected to result in a coherent change in microcirculatory blood flow, but this has not been investigated. This study investigated the effect of altering VA-ECMO pump flow rate on sublingual microcirculation.

Methods: Sublingual microcirculation images were recorded using an incident dark-field hand held vital microscope at two time points, within $24 \mathrm{~h}$ (T1) and at 24-48 h (T2) after VA-ECMO placement. Microcirculation was measured before and 5 minutes after the changes in VA-ECMO pump flow rate at each time point. Events of changing VA-ECMO pump flow rate at T1 and T2 were divided into four groups according to changes in perfused vessel density (PVD): Group A, increased pump flow rate and increased or sustained PVD; Group B, increased pump flow rate and decreased PVD; Group C, decreased pump flow rate and increased or sustained PVD; and Group D, decreased pump flow rate and decreased PVD. The microcirculatory parameters, clinical parameters, and prognosis of each subgroup were recorded. Clinical parameters on 14-day and 28-day survivors and non-survivors were also compared.

Results: A total of 25 patients were enrolled, and 38 events with good-quality images at T1 and T2 were categorized. Opposing response of microcirculation was observed in $43.5 \%$ of events when VA-ECMO pump flow rate was increased. Microcirculation was decreased in $33.4 \%$ of events when VA-ECMO pump flow rate was reduced. No predictive values in microcirculatory or macrocirculatory parameters before changing VA-ECMO pump flow rate were identified.

Conclusions: Changes in VA-ECMO pump flow rate may have opposing effects on sublingual microcirculatory functional capillary density. We suggest that tandem measurements of microcirculation before and after altering the VA-ECMO pump flow may help to ensure optimal tissue perfusion.

\section{Background}

Venoarterial Extracorporeal Membrane Oxygenation (VA-ECMO) has become a promising option for bridge support in patients with acute cardiopulmonary failure [1-3], including acute decompensated heart failure, myocardial infarction, pulmonary embolism, or extracorporeal cardiopulmonary resuscitation. A VA-ECMO pump flow rate that is too low leads to abnormal tissue perfusion and poor prognosis. In addition, pump flow-related complications such as kidney injury, cerebral stroke, and hemorrhage are associated with high morbidity and mortality [4, 5]. Neurological deficit is one of the most common complications of VA-ECMO placement, and flow optimization is essential to maintaining adequate tissue and brain perfusion [6]. The optimization of VA-ECMO pump flow is a challenge in clinical practice. 
Poor microcirculation parameters may reflect inadequate tissue perfusion [7, 8]. Our previous study and that conducted by Kara et al. revealed that a microcirculatory unresponsiveness to VA-ECMO in the initial hours predicted adverse outcomes $[9,10]$, and microcirculatory dysfunction can co-exist with normal macrocirculatory parameters [10]. Akin et al. identified an association between sustained microcirculation during reduction in VA-ECMO pump flow rate and successful weaning from VA-ECMO [11], but no study has reported the effects of changes in pump flow rate on microcirculation at early stage of VA-ECMO support. This study investigated the effects of changes in VA-ECMO pump flow rate on sublingual microcirculation within 24 and at 24-48 hours after VA-ECMO placement.

\section{Methods}

\section{Study design and patient selection}

This prospective observational study was approved by the Research Ethics Committee of National Taiwan University Hospital (approval number: 201703011RINA) and registered on the ClinicalTrials.gov protocol registration system (ID: NCT03210818). It was conducted at National Taiwan University Hospital between November 2017 and December 2018. Participants were selected from patients receiving ECMO support on the basis of eligibility screening conducted within 12 hours following ECMO placement. Patients who received VA-ECMO support and were between the ages of 20 and 90 years were included. Patients were excluded if they declined to participate, had received re-implantation of ECMO, died within 12 hours, or had circumstance that prevented sublingual microcirculation from being measured within 24 hours after initiating VA-ECMO, such as those in which placement occurred in the evening or the research nurse was on leave. Informed consent was obtained from patients' legally authorized representatives before study enrollment.

\section{VA-ECMO components and placement}

For all enrolled patients, the placement and principal components of the VA-ECMO were the same as described in our previous study [9]. To avoid possible malperfusion of the distal limb, an antegrade distal perfusion catheter was used when the mean pressure of the superficial femoral artery was below $50 \mathrm{~mm}$ $\mathrm{Hg}$ [12]. All patients received standard VA-ECMO management and routine intensive care unit (ICU) care. Heparin was continuously administered to maintain an activated clotting time of 160-180 s if no active bleeding or other complications were observed. The following data were recorded: age, gender, height, body weight, sequential organ failure assessment (SOFA) score [13], indications for VA-ECMO, VA-ECMO pump flow rate, heart rate, mean arterial pressure (MAP), lactate level, activated clotting time, hemoglobin level, fluid balance, and inotropic score. The inotropic score was calculated as $100 \times$ epinephrine dose $(\mathrm{mcg} / \mathrm{kg} / \mathrm{min})+100 \times$ norepinephrine dose $(\mathrm{mcg} / \mathrm{kg} / \mathrm{min})+$ dopamine dose $(\mathrm{mcg} / \mathrm{kg} / \mathrm{min})+$ dobutamine dose $(\mathrm{mcg} / \mathrm{kg} / \mathrm{min})$ [14]. The simultaneous use of intra-aortic balloon pump (IABP) with VA-ECMO support was recorded. The length of ICU and hospital stay, as well as survival status at 28 days were also recorded. 
The ECMO team adjusted the VA-ECMO pump flow rate to maintain an MAP $>60 \mathrm{~mm} \mathrm{Hg}$, central venous oxygen saturation $>70 \%$, central venous pressure $<15 \mathrm{mmHg}$, and lactate level of $<3 \mathrm{mmol} / \mathrm{L}$, and to avoid a urine output of $<0.5 \mathrm{~mL} / \mathrm{kg} / \mathrm{h}$, pulse pressure $<10 \mathrm{~mm} \mathrm{Hg}$, and ECMO-induced hemolysis, or to wean the patients off VA-ECMO support. Sublingual microcirculation images were recorded using an incident dark-field video microscope (CytoCam, Braedius Medical, Huizen, Netherlands) [15]. The images were recorded at two time points: within 24 hours (T1) and at 24 to 48 hours (T2) after VA-ECMO placement. At T1 and T2, images were recorded before changing VA-ECMO pump flow rate. After recording, the VA-ECMO pump flow rate was increased or reduced by the ECMO team member according to the treatment plan. The change in pump flow rate was recorded, and images were recorded at 5 minutes after the change.

\section{Measurements of sublingual microcirculation}

At each time point, five video sequences (length: $6 \mathrm{~s}$ each) were recorded at different sublingual sites and were digitally stored with code numbers to ensure the anonymity of patient information. Subsequent offline analyses were performed by a single observer blinded to patient information according to the international consensus guidelines for performing sublingual microcirculation by a Task Force of the European Society for Intensive Care Medicine [16]. Two or three sequences with appropriate image quality were selected for analysis using the semi-automated analysis software package Automated Vascular Analysis 3.0 [17].

In accordance with the afore mentioned consensus guidelines [16], the following parameters were investigated: (a) total vessel density (TVD; vessels less than $20 \mu \mathrm{m}$ ), (b) perfused vessel density (PVD), (c) proportion of perfused vessels (PPV), and (d) microvascular flow index (MFI) score. The software was used to automatically calculate TVD. The calculation of PVD was semiautomated using the procedure described in our previous study [9]. The MFI scores were semiquantitatively calculated according to the suggestions made at the roundtable conference [18].

\section{Grouping events of changes in VA-ECMO pump flow rate and changes in PVD}

Events of changing VA-ECMO pump flow rate at T1 and T2 were divided into the following four groups according to the changes in PVD. Group A included events of increased or sustained PVD after increasing the ECMO pump flow rate; Group B included events of reduced PVD after increasing the ECMO pump flow rate; Group $C$ included events of increased or sustained PVD after decreasing ECMO pump flow rate; and Group D included events of reduced PVD after decreasing ECMO pump flow rate.

\section{Statistical analysis}

All statistical analyses were performed using SPSS version 20 (IBM, Armonk, NY, USA). Normally distributed numerical data were expressed as means (standard deviation) and compared using t-tests. Nonnormal distribution of numerical data were expressed as medians (interquartile range) and compared using Mann-Whitney tests. Categorical variables were described as percentages and were compared 
using chi-square tests or Fisher's exact tests as appropriate. A $p$ value of $<0.05$ indicated a significant difference.

\section{Results}

\section{Patient distribution and characteristics}

A total of 70 patients receiving VA-ECMO were considered for inclusion in this trial (Figure 1). A total of 45 patients were excluded. The enrolled 25 patients had 50 events of changing VA-ECMO pump flow rate at T1 and T2. A total of 38 events with good quality of microcirculation images were enrolled, 19 events at T1 and 19 events at T2 (Figure 1). Detailed indications of individual patients regarding VAECMO applications, key variables, 14- and 28-day survival, survival to discharge, and clinical outcomes are listed in Table 1. Values for basic patient characteristics, indication for VA-ECMO application, IABP use, fluid status at each time point, ECMO pump flow rate, inotropic score, and T1 SOFA score of the 14and 28-day survivors and nonsurvivors are presented in Table 2. The T1 inotropic score was lower for the 14-day survivors than for the nonsurvivors. The T1 fluid balance, T2 ECMO initial pump flow rate, and T1 SOFA score were significantly lower for the 14-and 28-day survivors than for the nonsurvivors.

\section{Hemodynamic parameters and lab data at different time points}

Values for the hemodynamic parameters and lab data at T1 and T2 are presented in Table 3. At T1, central venous oxygen saturation level was lower in Group A than in Group B [73 (4) \% vs. 85 (7) \%, $p=$ 0.012], and changes in MAP is higher in Group A than in Group B [5 (4) vs. 0 (4) mm Hg, $p=0.048$ ]; but other hemodynamic parameters did not differ significantly between these groups. No statistical differences of hemodynamic parameters and lab data could be determined between Group C and D at T1. At T2, MFI score before the changes in VA-ECMO pump flow rate was lower in Group A than in Group B [1.8 (0.6) vs. $2.6(0.1), p=0.020]$, but the observed MAP, inotropic score, lactate level, and central venous oxygen saturation level did not differ significantly between Group A and B. Hemodynamic parameters and lab data between Group $C$ and D showed no significant differences at T2.

\section{Changes in microcirculation following change in ECMO pump flow rate}

PVD values and MFI scores before and after changes in ECMO pump flow rate in the four groups are presented in Figure 2. Of the 23 events of increased VA-ECMO pump flow rate, 13 (56.5\%) events showed increased or sustained microcirculation (Group $A$ ) and 10 (43.5\%) events showed reduced microcirculation (Group B). In the 15 events of decreased VA-ECMO pump flow rate, 10 (66.7\%) events showed increased microcirculation (Group C) and 5 (33.3\%) events showed reduced microcirculation (Group D). The relation of changes between VA-ECMO pump flow rate and PVD values are presented in Figure 3A. The relation of changes between VA-ECMO pump flow rate and MFI scores are presented in Figure 3B. Microcirculatory parameters at the baseline of each group are presented in Table 4. No 
significant differences in microcirculatory parameters before changes in VA-ECMO pump flow rate were observed between Group A and B or between Group C and D.

\section{Discussion}

We found divergent effects of changing the VA-ECMO pump flow rate on microcirculatory convective capacity as measured by PVD. Our results indicated that microcirculation was decreased in $43.5 \%$ of events when the VA-ECMO pump flow rate was increased and in $33.3 \%$ of events when the VA-ECMO pump flow rate was reduced. In Group B, PVD and MFI decreased after the VA-ECMO pump flow rate was increased, suggesting that an inappropriate VA-ECMO pump flow support impaired microcirculation. In addition, our results revealed that initial $\mathrm{ScvO}_{2}$ and changes in MAP were different in Group $\mathrm{A}$ and $\mathrm{B}$ within 24 hours after VA-ECMO placement. We suggest that further studies are required to investigate the predictability of initial values and delta changes of macrocirculatory and microcirculatory variables on changes in microcirculation following a change in VA-ECMO pump flow rate.

Our main finding was that in a significant portion of patients increasing VA-EMCO pump flow rate instead of increasing tissue perfusion as would be expected, the converse occurred and PVD decreased. Thus, increasing VA-ECMO pump flow may cause a paradoxical decrease in tissue perfusion. Two mechanisms may cause this condition. First, decreased cardiac preload caused by increased venous drainage reduces the venous return of blood flow to the right atrium. At the same time, cardiac afterload and vascular resistance could increase as a result of increased VA-ECMO arterial blood flow $[19,20]$. The decreased preload and increased afterload may reduce the patient's own cardiac output and sublingual microcirculation. Second, the blood mixing zone moves because of alteration in the proportion of VAECMO blood supply. According to the results of a simulation study conducted by Stevens et al.[21], brain perfusion is determined according to the balance between VA-ECMO pump flow rate and the patient's own cardiac output when the mixing zone is proximal to the aortic arch [21, 22]. If the patient's residual cardiac function fails to overcome the upcoming pressure work when the VA-ECMO pump flow rate increases, then the patient's own cardiac output will be offset. When the mixing zone moves closer to the aortic arch, the brain blood flow rate decreases. Brain hypoperfusion leads to reduced oxygen delivery and can significantly impair brain function and cause neurological complications. In a study conducted by Nasr et al., $4 \%$ of patients treated with VA-ECMO had an ischemic stroke, which led to a higher rate of discharge to long-term facilities, longer length of stay, and higher hospitalization costs [23]. Subsequent neurological complications, associated with poor outcomes and higher mortality, has become some of the main concerns during VA-ECMO support [24]. Measurement of sublingual microcirculation may provide an effective measure of whether this condition occurs. An animal study conducted by Boucek et al. revealed moderate correlations between cerebral regional oxygen saturation $\left(\mathrm{rSO}_{2}\right)$ and changes in microcirculatory parameters during cardiopulmonary resuscitation [25]. Moreover, brain $\mathrm{rSO}_{2}$ correlated 
well with global cerebral perfusion [25]. Tamosuitis et al. found that MFI scores were significantly lower for brain and sublingual mucosa of brain-dead patients compared with those of healthy controls [26]. Therefore, our results suggest that sublingual microcirculation may reflect brain perfusion when the VAECMO pump flow rate is being altered.

Another finding of our study is that microcirculation improved when the VA-ECMO pump flow rate was reduced. This may indicate that the patient's own cardiac output is sufficient and that the demand for VAECMO support is lower. Two possible mechanisms may contribute to this finding. First, a higher VA-ECMO pump flow rate causes a larger reduction in cardiac preload, stroke volume, and pulse pressure, and a larger increase in cardiac afterload [20]. This reminds us that a VA-ECMO pump flow rate that is too high may contribute to decreased cardiac output and left ventricular overload [27-29]. The second possible mechanism is recovery of the patient's cardiac function and the suitability of weaning from VA-ECMO. Patients in this condition who undergo prolonged VA-ECMO treatment are exposed to unnecessary risks of vascular complications, neurological complications, renal injury, bleeding, and infection [30]. Sublingual microcirculation monitoring may help to prevent excessively high VA-ECMO pump blood flow rate and accelerate the weaning process of VA-ECMO. In Group D, microcirculation decreased following the decrease in VA-ECMO pump flow rate, which may have indicated tissue hypoperfusion. Erroneous timing of weaning from VA-ECMO may contribute to this parlous situation. Microcirculation measurement may help to identify this situation and help ensure sufficient VA-ECMO pump flow.

Our study has several limitations. First, all patients received peripheral VA-ECMO. Mixing zone movement affects microcirculation on the basis of the physiological change resulted from peripheral VA-ECMO. This concept of a mixing zone may not be applicable for patients with central VA-ECMO. Second, this observational study had a small sample size, and there was no intention to construct a prediction model for determining changes in microcirculation following changes in VA-ECMO pump flow rate. Our results suggest that $\mathrm{ScVO}_{2}$ and MFI score before changes in pump flow rate and delta MAP after changes in pump flow rate might be investigated as predictors for responses of microcirculation to changes in VAECMO pump flow rate in further studies. Third, heterogeneous indications for VA-ECMO support make it difficult to interpret the association between changes in microcirculation and changes in VA-ECMO pump flow rate [31]. Future studies involving a larger number of patients with more homogeneous indications for VA-ECMO placement are required to better understand the association between changes in microcirculation and changes in VA-ECMO pump flow rate.

\section{Conclusions}

Our study revealed a high incidence of contradictory effects on microcirculation in response to changes in VA-ECMO pump flow rate. We suggest that tandem measurements of microcirculation before and after VA-ECMO pump flow change may help to ensure adequate tissue perfusion. Further studies are required to determine prediction factors for microcirculation change after changing the VA-ECMO pump flow rate.

\section{Abbreviations}


$\mathrm{HI}$

heterogeneity index; IABP:intra-aortic balloon pump; ICU:intensive care unit; MAP:mean arterial pressure; MFI:microvascular flow index; PPV:proportion of perfused vessels; PVD:perfused vessel density; SOFA:sequential organ failure assessment; TVD:total vessel density; VA-ECMO:venoarterial extracorporeal membrane oxygenation.

\section{Declarations}

\section{- Ethics approval and consent to participate}

This prospective observational study was approved by the Research Ethics Committee of National Taiwan University Hospital (approval number: 201703011RINA). Informed consents of the patients were obtained from their legally authorized representatives before enrollment in the study.

\section{- Consent for publication}

Not applicable.

\section{- Availability of data and material}

The datasets generated and/or analyzed during the current study are not publicly available due to the regulation of the Research Ethics Committee of National Taiwan University Hospital but are available from the corresponding author on reasonable request.

\section{- Competing interests}

We declare that we have no conflicts of interest in the authorship or publication of this contribution. Braedius Medical, a company owned by a relative of $\mathrm{Cl}$, has developed and designed a handheld microscope called CytoCam-IDF imaging. $\mathrm{Cl}$ has no financial relationship with Braedius Medical of any sort, i.e., never owned shares, or received consultancy or speaker fees from Braedius Medical. Active Medical of which $\mathrm{Cl}$ holds share runs an internet site called microcirculationacademy.org which offers educational courses and services related to clinical microcirculation.

\section{- Funding}

This work was supported, in part, by a grant from the Far Eastern Memorial Hospital and National Taiwan University Hospital Joint Research Program (106-FTN13) and a grant from the Taiwan Ministry of Science and Technology (MOST 107-2314-B-002-067-MY2).

\section{- Authors' contributions}

TJW contributed to the design of the study, patient enrollment, interpretation of the data, writing of the manuscript, and revision of the manuscript. CHW contributed to the design of the study, patient enrollment, interpretation of the data, and revision of the manuscript. YCY contributed to the 
interpretation of the data and revision of the manuscript. $\mathrm{CHL}$ contributed to the design of the study and patient enrollment. AC contributed to the design of the study. WSC contributed to interpretation of the results and revision of the manuscript. $\mathrm{CHH}$ contributed to the design of the study. MJW contributed to the design of the study. YJC contributed to the design of the study. YSC contributed to the design of the study and patient enrollment. TYL contributed to the design of the study. Cl contributed to the interpretation of the data and revision of the manuscript. YYC contributed to the design of the study, interpretation of the data, writing of the manuscript and revision of the manuscript.

\section{- Acknowledgements}

The authors thank all members of the ECMO team of National Taiwan University Hospital. They thank participants of the National Taiwan University Hospital Center of Microcirculation Medical Research (NCMMR), which includes Prof. Wei-Zen Sun, Dr. Chih-Min Liu, Dr. Po-Yuan Shih, Dr. Shih-Hong Chen, and Dr. Ching-Tang Chiu. They also thank Mr. Roger Lien (technician, MicroStar Instruments Co, Ltd, Taipei, Taiwan) and Ms. Yun-Ping Yang for technical assistance of microcirculation analysis. This manuscript was edited by Wallace Academic Editing

\section{References}

1. Makdisi G, Wang IW. Extra Corporeal Membrane Oxygenation (ECMO) review of a lifesaving technology. J Thorac Dis 2015, 7(7):E166-176.

2. Shekar K, Mullany DV, Thomson B, Ziegenfuss M, Platts DG, Fraser JF. Extracorporeal life support devices and strategies for management of acute cardiorespiratory failure in adult patients: a comprehensive review. Crit Care 2014, 18(3):219.

3. MacLaren G, Combes A, Bartlett RH. Contemporary extracorporeal membrane oxygenation for adult respiratory failure: life support in the new era. Intensive Care Med 2012, 38(2):210-220.

4. Pillai AK, Bhatti Z, Bosserman AJ, Mathew MC, Vaidehi K, Kalva SP. Management of vascular complications of extra-corporeal membrane oxygenation. Cardiovasc Diagn Ther 2018, 8(3):372-377.

5. Sidebotham D. Troubleshooting adult ECMO. J Extra Corpor Technol 2011, 43(1):P27-32.

6. Kazmi So, Sivakumar S, Karakitsos D, Alharthy A, Lazaridis C. Cerebral Pathophysiology in Extracorporeal Membrane Oxygenation: Pitfalls in Daily Clinical Management. Crit Care Res Pract 2018, 2018:3237810.

7. Ince $\mathrm{C}$. Hemodynamic coherence and the rationale for monitoring the microcirculation. Crit Care 2015, 19 Suppl 3:S8.

8. den Uil CA, Maat AP, Lagrand WK, van der Ent M, Jewbali LS, van Thiel RJ, et al. Mechanical circulatory support devices improve tissue perfusion in patients with end-stage heart failure or 
cardiogenic shock. J Heart Lung Transplant 2009, 28(9):906-911.

9. Yeh YC, Lee CT, Wang CH, Tu YK, Lai CH, Wang YC, et al. Investigation of microcirculation in patients with venoarterial extracorporeal membrane oxygenation life support. Crit Care 2018, 22(1):200.

10. Kara A, Akin S, Dos Reis Miranda D, Struijs A, Caliskan K, van Thiel RJ, et al. Microcirculatory assessment of patients under VA-ECMO. Crit Care 2016, 20(1):344.

11. Akin S, Dos Reis Miranda D, Caliskan K, Soliman Ol, Guven G, Struijs A, et al. Functional evaluation of sublingual microcirculation indicates successful weaning from VA-ECMO in cardiogenic shock. Crit Care 2017, 21(1):265.

12. Huang SC, Yu HY, Ko WJ, Chen YS. Pressure criterion for placement of distal perfusion catheter to prevent limb ischemia during adult extracorporeal life support. J Thorac Cardiovasc Surg 2004, 128(5):776-777.

13. Ferreira FL, Bota DP, Bross A, Melot C, Vincent JL. Serial evaluation of the SOFA score to predict outcome in critically ill patients. JAMA 2001, 286(14):1754-1758.

14. Shore S, Nelson DP, Pearl JM, Manning PB, Wong H, Shanley TP, et al. Usefulness of corticosteroid therapy in decreasing epinephrine requirements in critically ill infants with congenital heart disease. Am J Cardiol 2001, 88(5):591-594.

15. Milstein DMJ RE IC. A novel computer-controlled high resolution video microscopy imaging system enables measuring mucosal subsurface focal depth for rapid acquisition of oral microcirculation video images. Intensive Care Med 2012, 38:S271.

16. Ince C, Boerma EC, Cecconi M, De Backer D, Shapiro NI, Duranteau J, et al. Second consensus on the assessment of sublingual microcirculation in critically ill patients: results from a task force of the European Society of Intensive Care Medicine. Intensive Care Med 2018, 44(3):281-299.

17. Dobbe JG, Streekstra GJ, Atasever B, van Zijderveld R, Ince C. Measurement of functional microcirculatory geometry and velocity distributions using automated image analysis. Med Biol Eng Comput 2008, 46(7):659-670.

18. De Backer D HS, Boerma C, Goedhart P, Buchele G, Ospina-Tascon G, Dobbe I, et al. How to evaluate the microcirculation: report of a round table conference. . Critical care (London, England) 2007, 11(5):R101.

19. Baran DA. Extracorporeal Membrane Oxygenation (ECMO) and the Critical Cardiac Patient. Curr Transplant Rep 2017, 4(3):218-225.

20. Chung M, Shiloh AL, Carlese A. Monitoring of the adult patient on venoarterial extracorporeal membrane oxygenation. ScientificWorldJournal 2014, 2014:393258.

21. Stevens MC, Callaghan FM, Forrest P, Bannon PG, Grieve SM. Flow mixing during peripheral venoarterial extra corporeal membrane oxygenation - A simulation study. J Biomech 2017, 55:64-70.

22. Alwardt CM, Patel BM, Lowell A, Dobberpuhl J, Riley JB, DeValeria PA. Regional perfusion during venoarterial extracorporeal membrane oxygenation: a case report and educational modules on the concept of dual circulations. J Extra Corpor Technol 2013,45(3):187-194. 
23. Nasr DM, Rabinstein AA. Neurologic Complications of Extracorporeal Membrane Oxygenation. J Clin Neurol 2015, 11(4):383-389.

24. Le Guennec L, Cholet C, Huang F, Schmidt M, Brechot N, Hekimian G, et al. Ischemic and hemorrhagic brain injury during venoarterial-extracorporeal membrane oxygenation. Ann Intensive Care 2018, 8(1):129.

25. Boucek T, Mlcek M, Krupickova P, Huptych M, Belza T, Kittnar O, et al. Brain perfusion evaluated by regional tissue oxygenation as a possible quality indicator of ongoing cardiopulmonary resuscitation. An experimental porcine cardiac arrest study. Perfusion 2018, 33(1_suppl):65-70.

26. Tamosuitis T, Pranskunas A, Balciuniene N, Pilvinis V, Boerma EC. Conjunctival microcirculatory blood flow is altered but not abolished in brain dead patients: a prospective observational study. BMC Neurol 2016, 16:95.

27. Donker DW, Brodie D, Henriques JPS, Broome M. Left ventricular unloading during veno-arterial ECMO: a review of percutaneous and surgical unloading interventions. Perfusion 2019, 34(2):98-105.

28. Meani P, Gelsomino S, Natour E, Johnson DM, Rocca HB, Pappalardo F, et al. Modalities and Effects of Left Ventricle Unloading on Extracorporeal Life support: a Review of the Current Literature. Eur J Heart Fail 2017, 19 Suppl 2:84-91.

29. Fuhrman BP, Hernan LJ, Rotta AT, Heard CM, Rosenkranz ER. Pathophysiology of cardiac extracorporeal membrane oxygenation. Artif Organs 1999, 23(11):966-969.

30. Cheng R, Hachamovitch R, Kittleson M, Patel J, Arabia F, Moriguchi J, et al. Complications of extracorporeal membrane oxygenation for treatment of cardiogenic shock and cardiac arrest: a meta-analysis of 1,866 adult patients. Ann Thorac Surg 2014, 97(2):610-616.

31. Montero S, Chommeloux J, Franchineau G, Combes A, Schmidt M. Microcirculation in cardiogenic shock supported with extracorporeal membrane oxygenation: the need for a homogeneous population and strict evolution assessment. Crit Care 2018, 22(1):281.

\section{Tables}

Due to technical limitations, tables are only available as a download in the supplemental files section

\section{Figures}




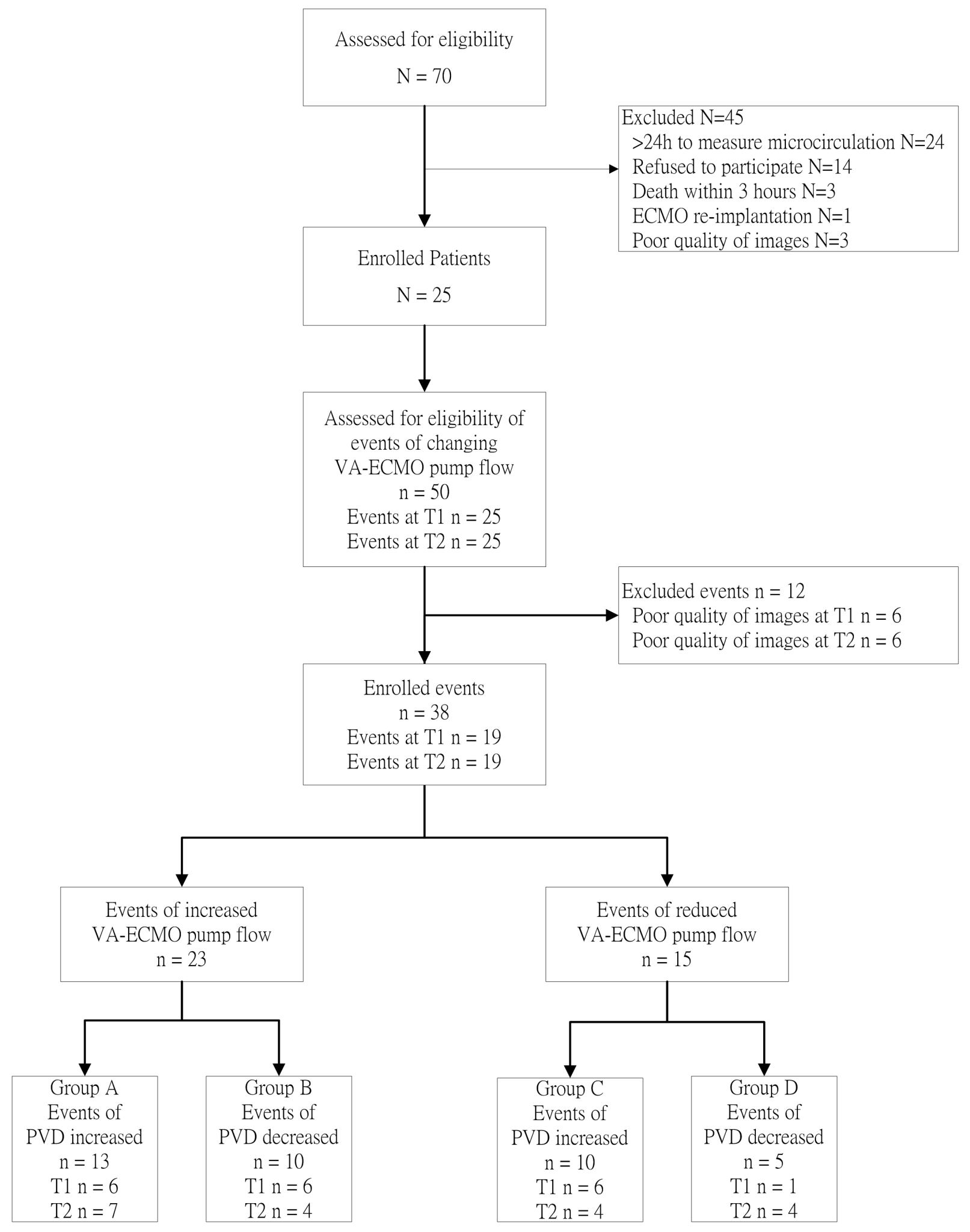

\section{Figure 2}

Consort flow chart of patients receiving venoarterial extracorporeal membrane oxygenation (VA-ECMO) life support PVD perfused vessel density, VA-ECMO venoarterial extracorporeal membrane oxygenation. T1: $<24$ hours after VA-ECMO initiation; T2: 24 to 48 hours after VA-ECMO initiation; N: number of patients, n: number of images. 
(A)

\section{Group A}

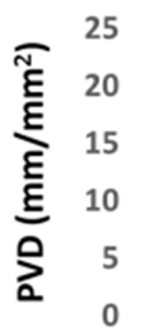

Before

Group A

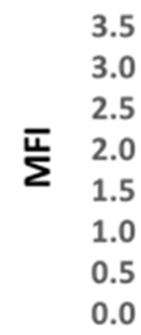

(B)

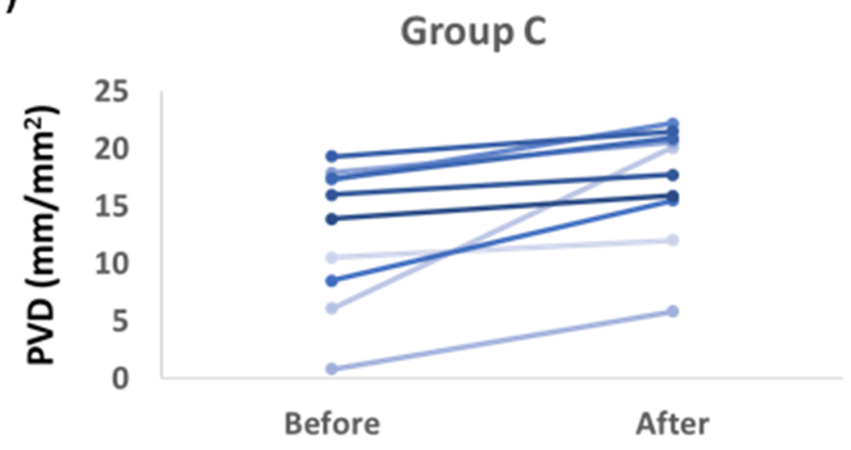

Group C

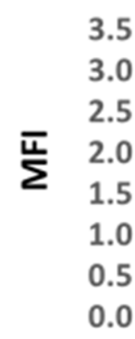

Group B

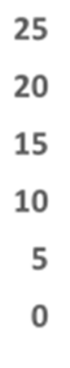

Before After

Group B

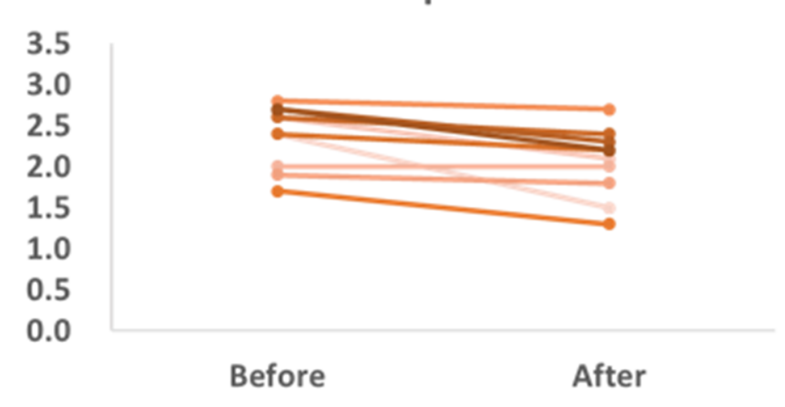

Group D

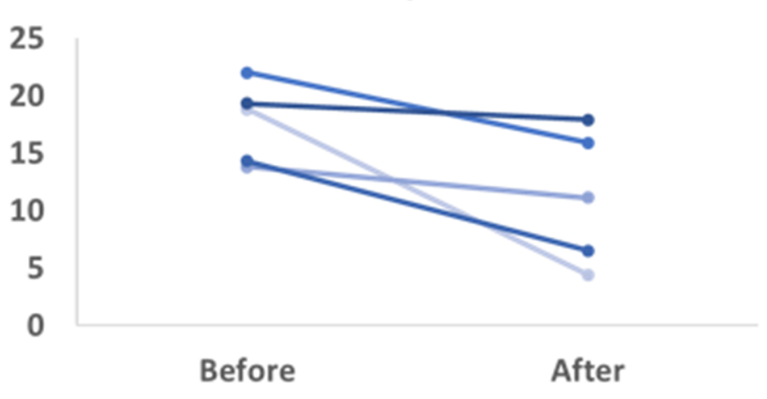

Group D

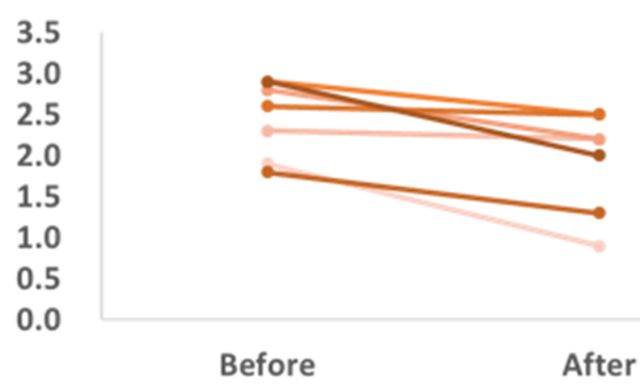

\section{Figure 3}

Effect of changes in venoarterial extracorporeal membrane oxygenation (VA-ECMO) pump flow rate on microcirculation (A) changes in PVD and MFI score in events of increased VA-ECMO pump flow rate. (B) changes in PVD and MFI score in events of decreased VA-ECMO pump flow rate. According to the effects of changing the VA-ECMO pump flow rate on microcirculation, events of changing VA-ECMO pump flow rate were categorized into Group A (increased pump flow rate, increased PVD), B (increased pump flow 
rate, decreased PVD), C (decreased pump flow rate, increased PVD), and D (decreased pump flow rate, decreased PVD). MFI microvascular flow index, PVD: perfused vessel density.

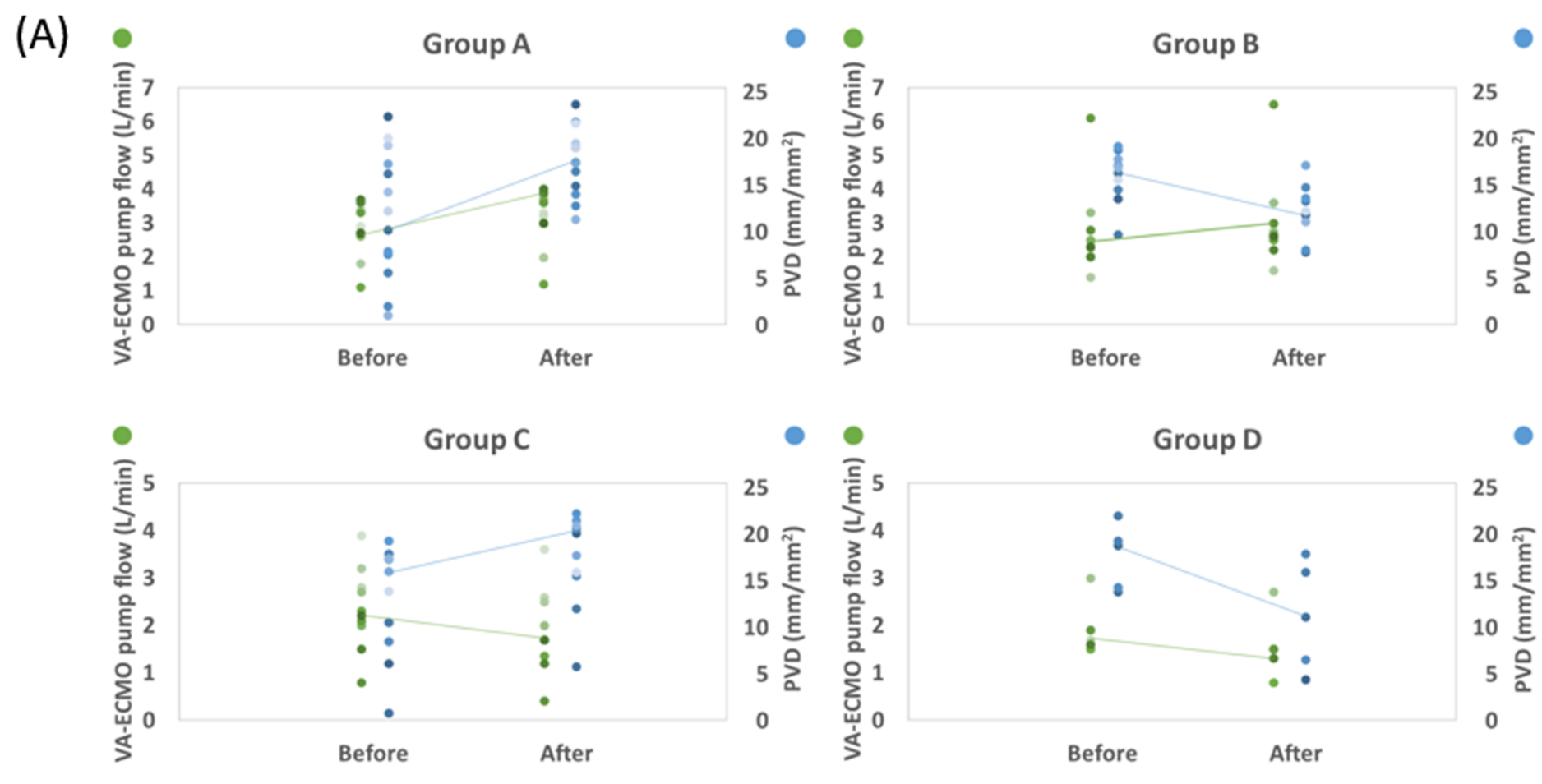

(B)
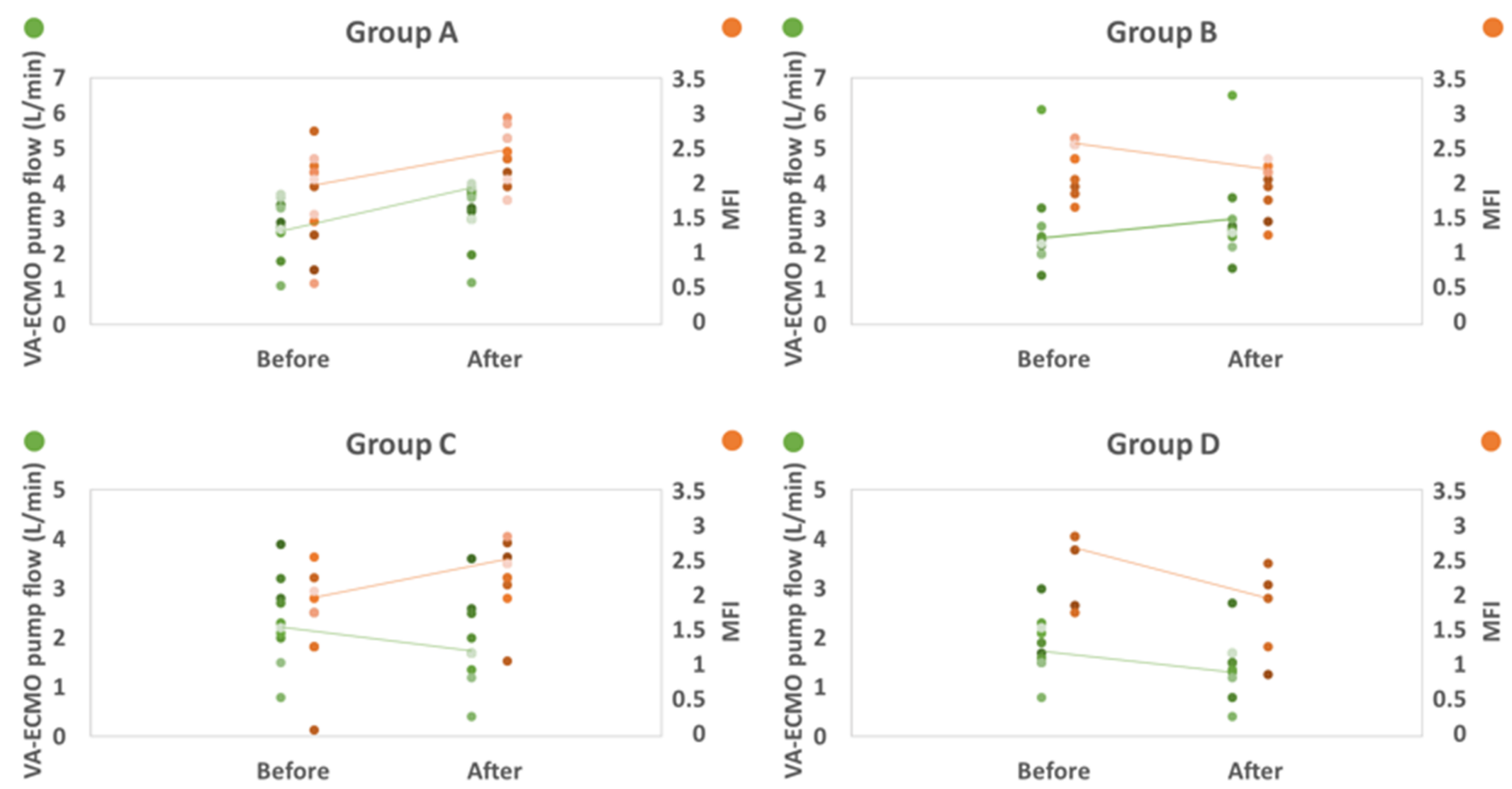

Figure 5

Relation of changes between venoarterial extracorporeal membrane oxygenation (VA-ECMO) pump flow rate and microcirculation (A) changes in VA-ECMO pump flow rate and PVD. (B) changes in VA-ECMO pump flow rate and MFI score. The lines represent changes of means in VA-ECMO pump flow rate, PVD, 
and MFI score. According to the effects of changing the VA-ECMO pump flow rate on microcirculation, events of changing VA-ECMO pump flow rate were categorized into Group A (increased pump flow rate, increased PVD), B (increased pump flow rate, decreased PVD), C (decreased pump flow rate, increased $P V D$ ), and D (decreased pump flow rate, decreased PVD). MFI microvascular flow index, PVD: perfused vessel density.

\section{Supplementary Files}

This is a list of supplementary files associated with this preprint. Click to download.

- Tables.docx

- Tables.docx 